\title{
Ball Control in High-speed Batting Motion using Hybrid Trajectory Generator
}

\author{
Taku Senoo Akio Namiki Masatoshi Ishikawa \\ Department of Information Physics and Computing, University of Tokyo \\ 7-3-1 Hongo, Bunkyo-ku, Tokyo 113-8656, Japan \\ Email: Taku_Seno@ipc.i.u-tokyo.ac.jp, Akio_Namiki@ipc.i.u-tokyo.ac.jp, Masatoshi_Ishikawa@ipc.i.u-tokyo.ac.jp
}

\begin{abstract}
Speeding up robot motion provides not only improvement in operating efficiency but also improves dexterous manipulation by taking advantage of an unstable state or noncontact state. In this paper we describe a hybrid trajectory generator that produces high-speed manipulation. This algorithm produces both mechanical high-speed motion and sensor-based reactive motion. As an example of high-speed manipulation, a robotic ball control in a batting task has been achieved. Performance evaluation is also analyzed.
\end{abstract}

\section{INTRODUCTION}

Human manipulation consists of not only precise slow motion but also dynamic motion such as "throwing", "hitting", "catching" and so on [1]. It is important for a robot system to be able to perform such motion in order to realize dexterous and flexible manipulation. One of the characteristics of such movement is that the robot be able to manipulate an object quickly. Here the important thing is for the robot to be aware that higher speed is required. At this time there are not many researchers working on speed of manipulation.

Kawamura et. al developed a high-speed manipulator FALCON based on a wire driven parallel mechanism [2]. It achieved peak accelerations of up to $43 \mathrm{G}$ and maximum velocities of $13 \mathrm{~m} / \mathrm{s}$. Kaneko et. al proposed the arm/gripper coupling mechanism where the spring energy accumulated in the arm is transferred to the kinetic energy of the arm [3]. It can achieve $100 \mathrm{G}$. In regard to speeding up the robot system, most researchers concentrate on the motor system.

In actual fact the ability to manipulate depends on a sensory function centered on visual or tactile perception, in addition to the motor function. However in most previous robot systems, the processing rate of vision sensors, for example, CCD $(30 \mathrm{~Hz})$ which is typical, is very much slower than the servo rate for robot control $(1 \mathrm{kHz})$. Therefore kinetic performance of a robot system is not dramatically increased by mechanical means alone. Moreover the faster a manipulator moves, the greater is the uncertainty. For these reasons, in order to robustly perform tasks requiring high-speed motion, real-time sensory feedback is necessary.

Namiki et al. developed a high-speed manipulation system through the introduction of high-speed vision sensing at a rate of $1 \mathrm{kHz}$ [4]. They verified that a real-time vision-based processing was able to execute not only fast servo control but also higher-level trajectory planning or task setting. In case of a robot system with high-speed sensors, it is useful to define the motion of a manipulator directly using sensor-based information. In this approach, however, the task is executed with little regard for the speed of motion [5]. To produce highspeed manipulation, it is necessary to consider both the speed of motion and the speed of response explicitly.

Based on this background, we proposed a hybrid trajectory generator as a motion strategy for a high-speed robot system and achieved a batting task [6]. This trajectory consists of both fast motion without depending on object information and tracking motion for target movement. In this paper, we extend the algorithm to control the direction of the ball after impact. In addition the dynamical analysis of a batting task is proposed.

\section{HYBRID TRAJECTORY AND ITS APPLICATION TO BATTING TASK}

\section{A. Concept}

Motion of a manipulator is produced mainly by two factors: planning a desired trajectory and designing a control system. There are many studies in which a complicated controller is designed in order to track any desired trajectory. In many cases this approach is useful. In the case of high-speed motion, however, it is very difficult to actuate a manipulator exactly only by devising a control law. This is because of the effect of nonlinear dynamics, parameter error and limit constraints of torques and angular velocities. Accordingly we focus on planning the trajectory rather than designing a control law. Our goal is to produce fast and smooth motion that is suitable for a robot to perform.

Therefore we define a desired trajectory in the joint coordinate space $\mathbb{R}^{n}$, where $n$ is the number of degrees of freedom of the manipulator, except for the collision condition expressed in the work space $\mathbb{R}^{6}$. In comparison with the trajectory generated in the work space, this has some advantages. For instance it is easy to generate a smooth trajectory, avoid singular points, and judge whether limit constraints are satisfied.

\section{B. Motion Strategy}

We describe a proposed algorithm using batting motion as the example. Based on the above mentioned concept, we present an algorithm of hybrid trajectory. This motion has two components: one is high-speed motion, and the other is reactive motion shown in Fig.1. 


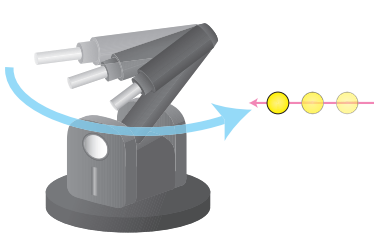

(a) SW mode

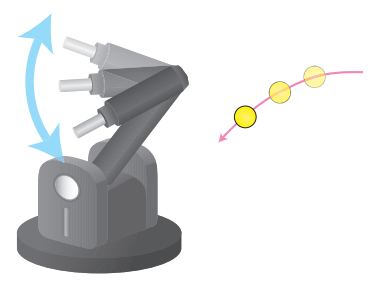

(b) HT mode
Fig. 1. Hybrid trajectory

The former swing motion is set to be high-speed motion so that its speed approaches the maximum. This function is represented by the time variable $t$. This motion does not depend on the target information except for the starting time of the swing. This motion is defined as SW mode.

The latter hitting motion is equivalent to mapping the trajectory of the manipulator to sensor information directly. Its trajectory is represented by the feature quantity of an object $\boldsymbol{\xi}$. In the case of the batting task, this means that the manipulator can hit a breaking ball in contrast with an approach based only prediction. This motion is defined as HT mode.

In addition it is necessary to introduce a varible that integrates the motion in the SW mode and the HT mode. Therefore a feature quantity $\boldsymbol{\eta}^{S W}$ that uniquely defines the SW mode motion uniquely is introduced. This means that the motion in the HT mode is modified by taking into consideration the motion in the SW mode. As a result, the vector of joint angles $\boldsymbol{q} \in \mathbb{R}^{n}$ is defined as

$$
\boldsymbol{q}=\boldsymbol{f}\left(\boldsymbol{\xi}, \boldsymbol{\eta}^{S W}, t\right) \in \mathbb{R}^{n} .
$$

Suppose that each motion is distributed for each degree of freedom, the joint vector is represented as

$$
\boldsymbol{q}=\left[\begin{array}{l}
\boldsymbol{q}^{S W}(t) \\
\boldsymbol{q}^{H T}\left(\boldsymbol{\xi}, \boldsymbol{\eta}^{S W}\right)
\end{array}\right]
$$

where $\boldsymbol{q}^{S W} \in \mathbb{R}^{n_{s}}, \boldsymbol{q}^{H T} \in \mathbb{R}^{n_{t}}$ means the variable corresponding to each mode. In addition $n_{s}, n_{t}$ represents the degrees of freedom of each mode and they satisfy $n_{s}+n_{t}=n$.

In this paper, we adopt a fifth order polynomial as a trajectory function in order to continuously control position, velocity, and acceleration:

$$
\boldsymbol{q}=\sum_{i=0}^{5} \boldsymbol{k}_{i} t^{i}
$$

Moreover we select a target position $\boldsymbol{p}_{o} \in \mathbb{R}^{3}$ as target information $\boldsymbol{\xi}$, and time variabe $t$ as a feature quantity of SW mode $\boldsymbol{\eta}^{S W}$. Then the coefficient of trajectory $\boldsymbol{k}_{i} \in \mathbb{R}^{n}$ is represented as

$$
\boldsymbol{k}_{i}=\left[\begin{array}{l}
\boldsymbol{k}_{i}^{S W} \\
\boldsymbol{k}_{i}^{H T}\left(\boldsymbol{p}_{o}\right)
\end{array}\right], \quad \boldsymbol{k}_{i}^{S W}=\text { constant } .
$$

That is, the trajectory of a manipulator is determined by the coefficient $\boldsymbol{k}_{i}$.

To increase the velocity of an end-effector in serial link mechanism, it is necessary to speed up the velocity of joints

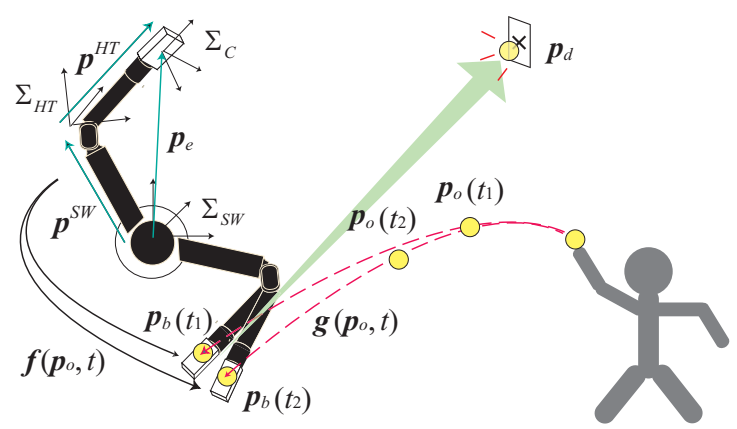

Fig. 2. Ball control in batting motion

on the bottom side. Besides it is easy to modify the trajectory of joints on the top side so that the manipulator can follow unpredictable objects during the swing. This is because the inertia of the end-effector is lower than the inertia of the bottom part. For this reason motion int the SW mode is assigned to the bottom side, and motion in the HT mode is assigned to the top side. Then the position of end-effector $\boldsymbol{p}_{e} \in \mathbb{R}^{3}$ is represented as

$$
\boldsymbol{p}^{S W}+{ }^{S} T_{H} \boldsymbol{p}^{H T}=\boldsymbol{p}_{e},
$$

where $\boldsymbol{p}^{S W} \in \mathbb{R}^{3}$ is represented in the coordinate $\Sigma_{S W}$ fixed to the 0 -th link (standard coordinates), $\boldsymbol{p}^{H T} \in \mathbb{R}^{3}$ is represented in the coordinate $\Sigma_{H T}$ fixed to the $n_{s}$-th link, and ${ }^{S} T_{H}\left(\boldsymbol{q}^{S W}\right) \in$ $\mathbb{R}^{3 \times 3}$ is a rotation matrix from $\Sigma_{H T}$ to $\Sigma_{S W}$ shown in Fig.2. Suppose that $l$ represents direct kinematics, the vector of the end-effector $\boldsymbol{r}_{e}^{T}=\left[\begin{array}{ll}\boldsymbol{p}_{e}^{T} & \phi_{e}^{T}\end{array}\right] \in \mathbb{R}^{6}$ is represnted as

$$
\boldsymbol{r}_{e}=\boldsymbol{l}(\boldsymbol{q}),
$$

where $\phi_{e} \in \mathbb{R}^{3}$ represents the posture of the end-effector.

\section{Batting Algorithm}

The trajectory of a manipulator is determined by following three steps.

( i ) sensing position of a target $\boldsymbol{p}_{o}$

(ii) generation of batting position and posture $\hat{\boldsymbol{r}}_{b}(t) \in \mathbb{R}^{6}$

(iii) determination of the trajectory $\boldsymbol{q}$ satisfying the boundary condition

Every cycle time of $1 \mathrm{~ms}$, the steps ( i ) and (ii) are repeated from the time the vision sensor recognizes a target until the start time of the swing $t=0$, and all steps ( i ), (ii) and (iii) are repeated until the time of hitting $t=t_{b}$. This means that the hitting point is estimated before impact explicitly and the batting task is accomplished by modification of this point by visual feedback. The position and posture of the manipulator at impact point is expressed as $\boldsymbol{r}_{b}^{T}=\left[\begin{array}{ll}\boldsymbol{p}_{b}^{T} & \boldsymbol{\phi}_{b}^{T}\end{array}\right] \in \mathbb{R}^{6}$, and superscript ${ }^{\wedge}$ represents the estimate value.

Each step is described as follows.

( i ) Three dimensional visual information is calculated by means of stereovision with two active visions. By image-based visual servoing, the active vision executes $3 \mathrm{D}$ tracking so as to keep the target within the field of view. The first order moment of the image is treated as a position of the target. 
(ii) The position and posture of the manipulator at the time of hitting is determined in this step.

1) position: Using a function $g$, we set the hitting point as $\boldsymbol{p}_{b}(t)=\boldsymbol{g}\left(\boldsymbol{p}_{o}, t\right)$. This function $\boldsymbol{g}$ is set so the ball will collide with the bat at $t=t_{b}$ and it has to satisfy following the formula:

$$
\boldsymbol{g}\left(\boldsymbol{p}_{o}, t_{b}\right)=\boldsymbol{p}_{o}\left(t_{b}\right)=\boldsymbol{p}_{e}\left(t_{b}\right) .
$$

In this experiment, the predicted trajectory is adopted as the function $g$ by fitting a function using successive least-squares estimations.

The manipulator starts to swing at the moment the hitting point $\hat{\boldsymbol{p}}_{b}$ enters the strike zone. In IV-B, we give more detailed information about the strike zone, which is a curved surface giving consideration to the dynamics of the manipulator. We assume that at the time of hitting the ball reaches the following plane:

$$
x=\hat{\boldsymbol{p}}_{b}(0) \cdot \boldsymbol{e}_{x},
$$

where $\boldsymbol{e}_{x} \in \mathbb{R}^{3}$ represents the $x$-axis unit vector, whose direction is the throwing direction. The initial estimated hitting point is on this plane. After the start time of the swing, we set the hitting point to the intersection between the predicted trajectory of the ball and the plane expressed by Eq.(8).

2) posture: Let us consider the impact modeling between a flat bat and a spherical ball in order to spray hit. We assume that the manipulator is controlled rigidly, and is not affected by the impact force. In addition we ignore the impulse of gravity at the time of hitting. We set $\boldsymbol{v}, \boldsymbol{v}^{\prime} \in \mathbb{R}^{3}$ as the velocity of the ball before and after collision respectively, and similarly $\boldsymbol{\omega}, \boldsymbol{\omega}^{\prime} \in \mathbb{R}^{3}$ represents the angular velocity. The law of conservation of linear momentum and angular momentum is given as

$$
\begin{aligned}
m\left(\boldsymbol{v}^{\prime}-\boldsymbol{v}\right) & =\overline{\boldsymbol{F}}+\overline{\boldsymbol{R}} \\
I\left(\boldsymbol{\omega}^{\prime}-\boldsymbol{\omega}\right) & =\boldsymbol{d} \times \overline{\boldsymbol{F}},
\end{aligned}
$$

where $m$ is the mass of the ball, $I=\frac{2}{5} m a^{2}$ is the moment of inertia, $\boldsymbol{d} \in \mathbb{R}^{3}$ is a vector directed toward the center of mass from the contact point, $a=\|\boldsymbol{d}\|$ is the radius of the ball, and $\overline{\boldsymbol{F}}, \overline{\boldsymbol{R}} \in \mathbb{R}^{3}$ is the impulse of frictional force and normal force respectively. These variables are described in the coordinate $\Sigma_{C}$, whose origin is fixed at the surface of the bat. Moreover the bounce equation without sliding is expressed as

$$
\boldsymbol{v}^{\prime}=\boldsymbol{\omega}^{\prime} \times \boldsymbol{d}-e \frac{\boldsymbol{v} \cdot \boldsymbol{d}}{\|\boldsymbol{d}\|^{2}} \boldsymbol{d},
$$

where $e$ is the coefficient of restitution. Suppose that the normal direction of the bat is set as the $z$-axis of the coordinate $\Sigma_{C}$. Using Eqs.(9) (11), the rebound velocity is calculated as

$$
\begin{aligned}
v_{x}^{\prime} & =\frac{2}{7}\left(a \omega_{y}+\frac{5}{2} v_{x}\right) \\
v_{y}^{\prime} & =\frac{2}{7}\left(-a \omega_{x}+\frac{5}{2} v_{y}\right) \\
v_{z}^{\prime} & =-e v_{z} .
\end{aligned}
$$

We used a ball with radius $a=0.05 \mathrm{~m}$, so the the angular velocity of right side influences only a fiftieth part of the velocity before impact. Accordingly the rebound velocity is approximated by $\boldsymbol{v}^{\prime}=K \boldsymbol{v}, K=\operatorname{diag}\left(\frac{5}{7}, \frac{5}{7},-e\right)$. We assume that the target continues in a state of uniform motion after impact. Then in order to control the direction of the ball after hitting, the posture of a manipulator is calculated so that the following equation is satisfied:

$$
{ }^{S} T_{C} K \boldsymbol{v} \times\left(\boldsymbol{p}_{d}-\boldsymbol{p}_{b}\right)=\mathbf{0},
$$

where $\boldsymbol{p}_{d} \in \mathbb{R}^{3}$ is an objective point and ${ }^{S} T_{C}(\boldsymbol{q}) \in \mathbb{R}^{3 \times 3}$ is a rotation matrix from $\Sigma_{C}$ to $\Sigma_{S W}$. The posture $\phi_{b}$ can be calculated by Eq.(13).

(iii) The boundary conditions are written as

$$
\boldsymbol{q}(0)=\boldsymbol{q}_{s}, \quad \boldsymbol{q}\left(t_{b}\right)=\boldsymbol{l}^{-1}\left(\boldsymbol{r}_{b}\right)=\boldsymbol{q}_{b},
$$

where $\boldsymbol{q}_{s} \in \mathbb{R}^{n}$ are initial joints and $\boldsymbol{q}_{b} \in \mathbb{R}^{n}$ are joints at the time of hitting. The coefficient of the trajectory $\boldsymbol{k}_{i}$ is transformed into the following expression:

$$
\begin{aligned}
& \boldsymbol{k}_{0}= \boldsymbol{q}_{s}, \quad \boldsymbol{k}_{1}=\dot{\boldsymbol{q}}(0), \quad \boldsymbol{k}_{2}=\frac{1}{2} \ddot{\boldsymbol{q}}(0) \\
& \boldsymbol{k}_{3}= \frac{1}{2 t_{b}^{3}}\left\{20\left[\boldsymbol{q}_{b}-\boldsymbol{q}_{s}\right]-t_{b}\left[8 \boldsymbol{c}_{v}+12 \dot{\boldsymbol{q}}(0)\right]\right. \\
&\left.\quad+t_{b}^{2}\left[\boldsymbol{c}_{a}-3 \ddot{\boldsymbol{q}}(0)\right]\right\} \\
& \boldsymbol{k}_{4}= \frac{1}{2 t_{b}^{4}}\left\{-30\left[\boldsymbol{q}_{b}-\boldsymbol{q}_{s}\right]+t_{b}\left[14 \boldsymbol{c}_{v}+16 \dot{\boldsymbol{q}}(0)\right]\right. \\
&\left.\quad-t_{b}^{2}\left[2 \boldsymbol{c}_{a}-3 \ddot{\boldsymbol{q}}(0)\right]\right\} \\
& \boldsymbol{k}_{5}= \frac{1}{2 t_{b}^{5}}\left\{12\left[\boldsymbol{q}_{b}-\boldsymbol{q}_{s}\right]-6 t_{b}\left[\boldsymbol{c}_{v}+\dot{\boldsymbol{q}}(0)\right]\right. \\
&\left.\quad+t_{b}^{2}\left[\boldsymbol{c}_{a}-\ddot{\boldsymbol{q}}(0)\right]\right\},
\end{aligned}
$$

where $\boldsymbol{c}_{v}, \boldsymbol{c}_{a}$ represents an arbitrary vector. If the hitting point and swing time are constants during the swing, the vector is represented by $\boldsymbol{c}_{v}=\dot{\boldsymbol{q}}\left(t_{b}\right), \boldsymbol{c}_{a}=\ddot{\boldsymbol{q}}\left(t_{b}\right)$ respectively. Thus we can set a rough speed of the manipulator at impact point by adjusting the arbitrary vector.

The vector $\boldsymbol{q}_{b}^{S W}$ is defined as

$$
\boldsymbol{q}_{b}^{S W}=A \boldsymbol{l}^{-1}\left(\hat{\boldsymbol{r}}_{b}(0)\right),
$$

where $A=\left[\begin{array}{ll}E_{n_{s}} & 0\end{array}\right] \in \mathbb{R}^{n_{s} \times n}$, and $E_{n_{s}} \in \mathbb{R}^{n_{s} \times n_{s}}$ is a unit matrix. The $\boldsymbol{k}_{i}^{S W}$ is calculated when the $\boldsymbol{q}_{b}^{S W}$ is substituted for Eq.(15). We adjust $\boldsymbol{c}_{v}, \boldsymbol{c}_{a}$ so that the velocity or torque is as high as possible within the output limit. Therefore the highspeed motion can be realized. Since the $\boldsymbol{k}_{i}^{S W}$ is calculated by the estimated hitting point $\hat{\boldsymbol{r}}_{b}(0)$ in each trial, it is determined as an appropriate value for the random ball.

Once the manipulator starts to swing, the trajectory must be generated taking into consideration to the motion in the SW mode. Using Eqs.(5) and (13), the vector $\boldsymbol{q}_{b}^{H T}$ is computed so that the following is satisfied:

$$
\begin{aligned}
& \boldsymbol{l}_{H T}\left(\boldsymbol{q}_{b}^{H T}\right)={ }^{S} \hat{T}_{H}^{-1}\left(\hat{\boldsymbol{p}}_{b}(t)-\hat{\boldsymbol{p}}^{S W}\right) \\
& { }^{H} \hat{T}_{C}\left(\boldsymbol{q}_{b}^{H T}\right)=\gamma^{S} \hat{T}_{H}^{-1}\left(\boldsymbol{p}_{d}-\hat{\boldsymbol{p}}_{b}(t)\right)(K \hat{\boldsymbol{v}})^{+},
\end{aligned}
$$

where $\boldsymbol{l}_{H T}$ represents direct kinematics of the HT mode satisfying $\boldsymbol{p}^{H T}=\boldsymbol{l}_{H T}\left(\boldsymbol{q}^{H T}\right), \gamma$ is a normalization constant, and ${ }^{H} T_{C}\left(\boldsymbol{q}^{H T}\right) \in \mathbb{R}^{3 \times 3}$ is a rotation matrix satisfying ${ }^{S} T_{C}=$ ${ }^{S} T_{H}{ }^{H} T_{C}$. In addition a suffix ${ }^{+}$represents a pseudo-inverse 


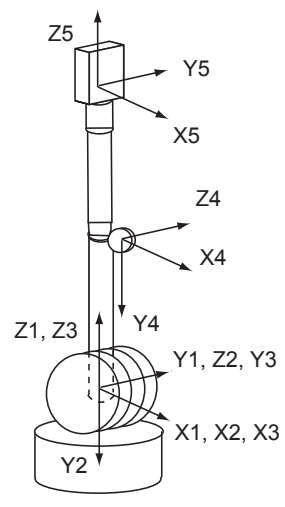

(a) Kinematics

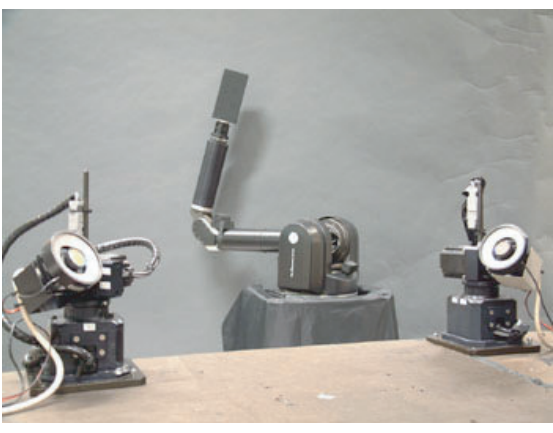

(b) Manipulator and vision system
Fig. 3. High-speed robot system

matrix. The $\boldsymbol{k}_{i}^{H T}$ is calculated when the $\boldsymbol{q}_{b}^{H T}$ is substituted for Eq.(15).

\section{EXPERIMENT}

\section{A. System Configuraiton}

The vision system consists of the 2-DOF (tilt and pan) active vision which is a column parallel vision system (CPV) [7]. The CPV has $128 \times 128$ pixel photo detectors and an all pixel parallel processing array. Various visual processing (moment detection, segmentation and so on) are achieved within $1 \mathrm{~ms}$ because execution is in parallel.

The kinetic system consists of a wire-drive manipulator (Barrett Technology Inc.). The kinematics of the manipulator is shown in Fig.3(a). The manipulator has 5-DOF consisting of revolution and bending motion alternately. High-speed movement with maximum velocity of the end-effector of $8 \mathrm{~m} / \mathrm{s}$ and maximum acceleration of $58 \mathrm{~m} / \mathrm{s}^{2}$ is achieved.

The cycle time of visual and control processing is set at $1 \mathrm{~ms}$. Figure3(b) shows the high-speed robot system.

\section{B. Experimental Setting}

A human threw a styrofoam ball with radius $5 \mathrm{~cm}$ towards the manipulator from $2.3 \mathrm{~m}$ distance. The manipulator hit the ball towards an objective point $\boldsymbol{p}_{d}=\left[\begin{array}{lll}1.9 & 0.0 & 1.3\end{array}\right]^{T}$. We set the second joint $q_{2}$ is fixed during the swing. When one joint is assigned to the motion in SW mode, three joints are assigned to the motion in HT mode. In addition we adopted a PD controller. Both before the impact and after the impact, the trajectory of the manipulator after was generated by a fifth order polynomial. This allows the manipulator to stop smoothly. As for other parameters, we set $\dot{\boldsymbol{q}}(0)=\ddot{\boldsymbol{q}}(0)=\mathbf{0}$, the time until hitting as $t_{b}=0.25 \mathrm{~s}$, and the whole swing time as $0.85 \mathrm{~s}$.

\section{Experimental Result}

From the moment the vision system finds the ball it takes $0.3 \sim 0.4 \mathrm{~s}$ for the ball to reach the hitting point. In this experiment, the batting task was achieved under the condition that the manipulator must immediately start to swing $0.1 \mathrm{~s}$ after the vision sensor recognizes the ball. The velocity of the ball

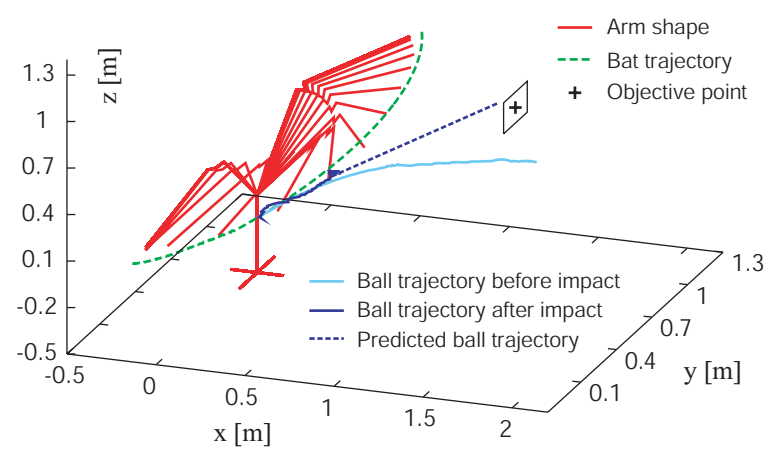

Fig. 4. Batting motion

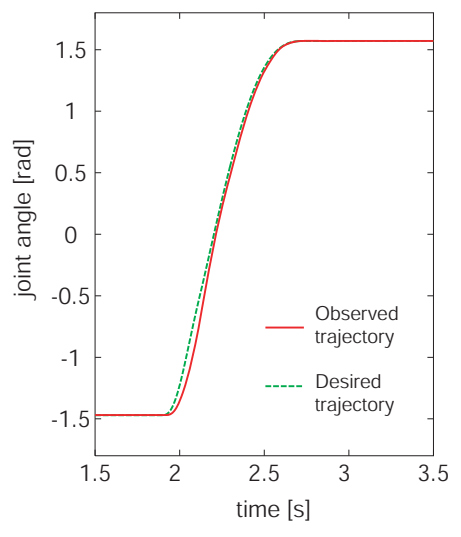

(a) SW mode

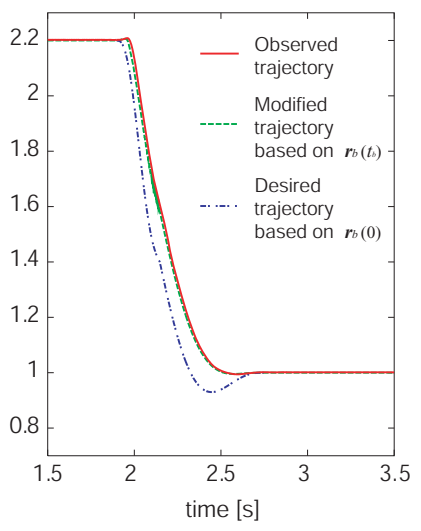

(b) HT mode
Fig. 5. Time response of joint angles

is $6 \sim 8 \mathrm{~m} / \mathrm{s}$, and the velocity of the end-effector is about $6 \mathrm{~m} / \mathrm{s}$ at impact point.

Figure 4 shows the motion of the arm and the ball. The ball is recognized at $x=2.1 \mathrm{~m}$ and is hit on the hitting point at $x=0.33 \mathrm{~m}$. From the data for ball position after hitting, it turns out that the hit ball heads in the direction to the objective point.

The time response of joint angles is shown in Fig.5. It turns out that the smooth joint trajectory is generated in either mode. In HT mode, the desired trajectory based on $\boldsymbol{r}_{b}(0)$ is modified to the one based on $\boldsymbol{r}_{b}\left(t_{b}\right)$ due to a shift of hitting point. Then the observed trajectory of the manipulator follows it. This result means that the manipulator can hit a breaking ball.

In this way, even though we use a simple controller, the manipulator achieves high-speed and reactive motion because of the appropriate desired command. In Fig.6 and Fig.7, the batting motion is shown as a continuous sequence of pictures taken at intervals of $132 \mathrm{~ms}$ and $30 \mathrm{~ms}$ respectively. The success rate was about $40 \%$. These experimental results are shown as a movie on the web site [8].

\section{ANALYSIS}

Kinematic and dynamic constraints of the manipulator is described with

$$
\boldsymbol{q}_{\min } \leq \boldsymbol{q} \leq \boldsymbol{q}_{\max }, \boldsymbol{\omega}_{\min } \leq \boldsymbol{\omega} \leq \boldsymbol{\omega}_{\max }, \boldsymbol{\tau}_{\min } \leq \boldsymbol{\tau} \leq \boldsymbol{\tau}_{\max },
$$



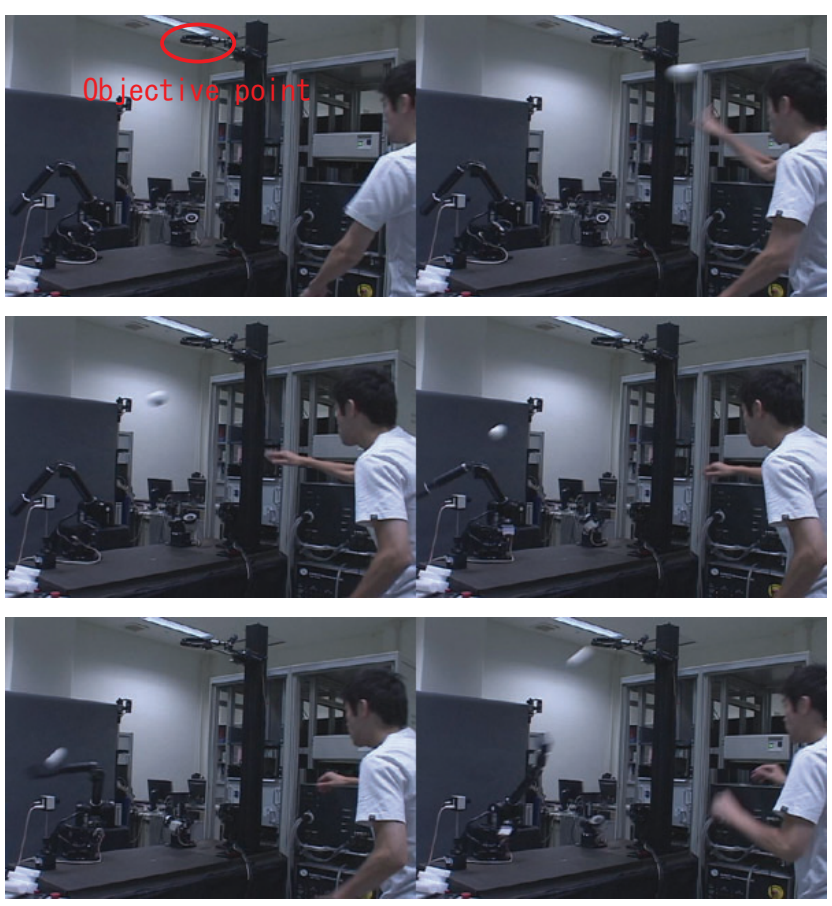

Fig. 6. Continuous sequence of pictures
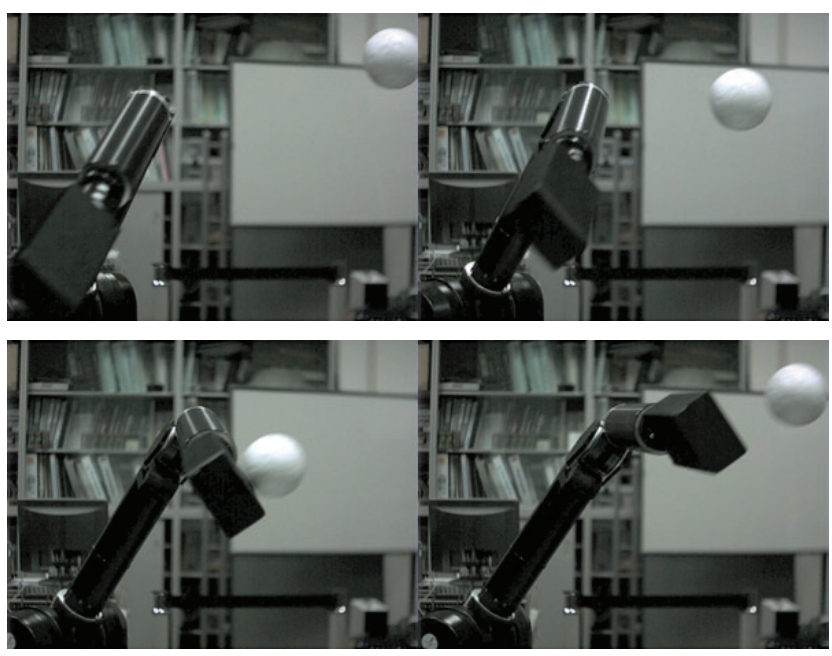

Fig. 7. Continuous sequence of pictures (close view)

where $\boldsymbol{\omega} \in \mathbb{R}^{n}$ is a vector for angular velocities, $\boldsymbol{\tau} \in \mathbb{R}^{n}$ is a vector for joint torques, and a suffix max, min means the maximum and minimum of the variable respectively. The angular velocity and the torque are computed as

$$
\boldsymbol{\omega}=\dot{\boldsymbol{q}}, \quad \boldsymbol{\tau}=\boldsymbol{M}(\boldsymbol{q}) \ddot{\boldsymbol{q}}+\boldsymbol{h}(\boldsymbol{q}, \dot{\boldsymbol{q}})+\boldsymbol{g}(\boldsymbol{q}),
$$

where $\boldsymbol{M}(\boldsymbol{q}) \in \mathbb{R}^{n \times n}$ is the inertia matrix, $\boldsymbol{h}(\boldsymbol{q}, \dot{\boldsymbol{q}}) \in \mathbb{R}^{n}$ is the coriolis and centrifugal force terms, and $\boldsymbol{g}(\boldsymbol{q}) \in \mathbb{R}^{n}$ is the gravity term.

Based on the dynamics of the manipulator and the constraints, the proposed trajectory is analyzed by computer simulation as follows.

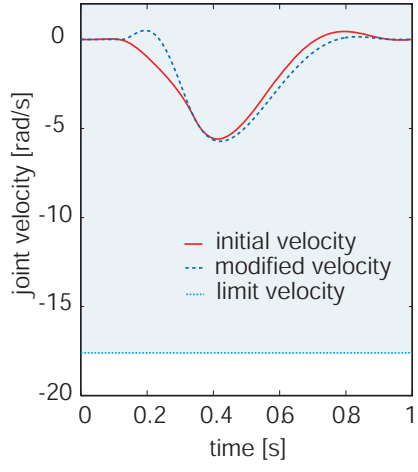

(a) Velocity response

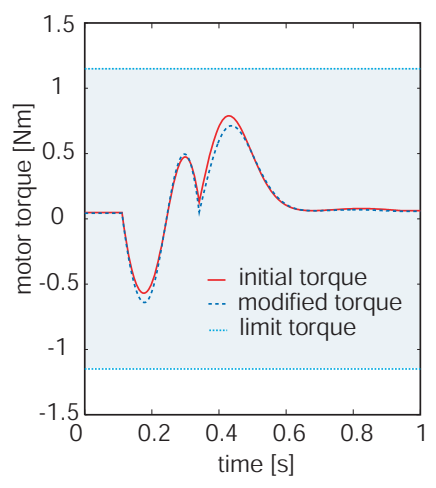

(b) Torque response
Fig. 8. Time response of velocity and torque

\section{A. Manipulator Trajectory}

Figure8 shows the time response of the joint velocity and a motor torque when the hitting point moves $10 \mathrm{~cm}$ during a swing. Even in this case, a smooth trajectory is generated similarly to the trajectory based on a fixed hitting point. The trajectory of the torque is changed continuously, but not smoothly at the start time of the swing and time of hitting due to the use of a fifth order polynomial and switching the trajectory at that time. This problem is solved by generating a function with consideration to the jerk. In addition a certain level of margin for torque is given so that the torque does not exceed the limit for the shift in hitting point or the effect of parameter errors.

\section{B. Strike Zone}

In our experimental setting, the area $S$ where the position of the end-effector is reached is expressed as

$$
\begin{aligned}
& S=\left\{\boldsymbol{p} \mid\left\|\boldsymbol{p}-\boldsymbol{p}^{\prime}\right\|=L_{u} \sin q_{2}, \boldsymbol{p}^{\prime} \in C\right\} \\
& C=\left\{\boldsymbol{p} \mid\|\boldsymbol{p}-\boldsymbol{\beta}\|=L_{f}, p_{x}=0\right\},
\end{aligned}
$$

where $L_{u}=\left\|\boldsymbol{p}^{S W}\right\|, L_{f}=\left\|\boldsymbol{p}^{H T}\right\|$ represents the length of the upper arm and front arm respectively and $\boldsymbol{\beta}=\left[\begin{array}{llll}0 & 0 & L_{u} \cos & q_{2}\end{array}\right]^{T}$. We define the strike zone as part of the area on the curved surface $S$, where the dynamics and constraints are considered. The hitting point at the start time of the swing $\hat{\boldsymbol{r}}_{b}(0)$ is determined as the intersection between this zone and the predicted trajectory of the ball.

Figure9 represents the strike zone. Viewed from the pitching direction, its height is $100 \mathrm{~cm}$ and width is $60 \mathrm{~cm}$. The strike zone depends not on the trajectory of the ball, but on the initial position of the arm and swing time. Since the manipulator cannot hit a ball that passes outside of the strike zone, we set the manipulator so that it does not swing at such a ball. In the case where $\hat{\boldsymbol{r}}_{b}(t)=$ constant during the swing, the task is achieved definitely in terms of the dynamics of the manipulator.

In the case of the batting task, there is a trade-off between shortening the swing time and expanding the strike zone. In this experiment, first we set the strike zone sufficient for the batting task, then we set the swing time at $0.25 \mathrm{~s}$ so that it is 


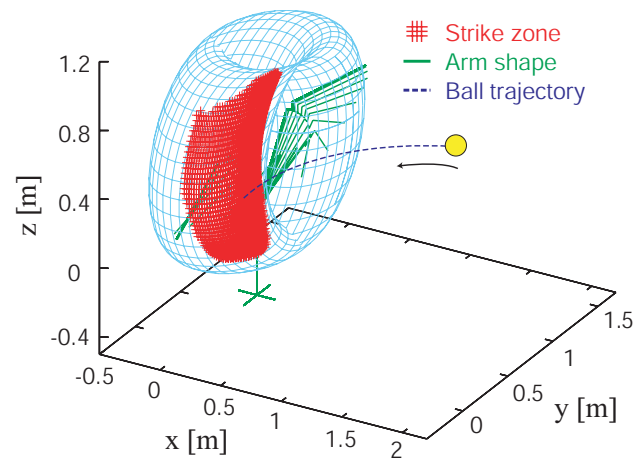

Fig. 9. Strike zone
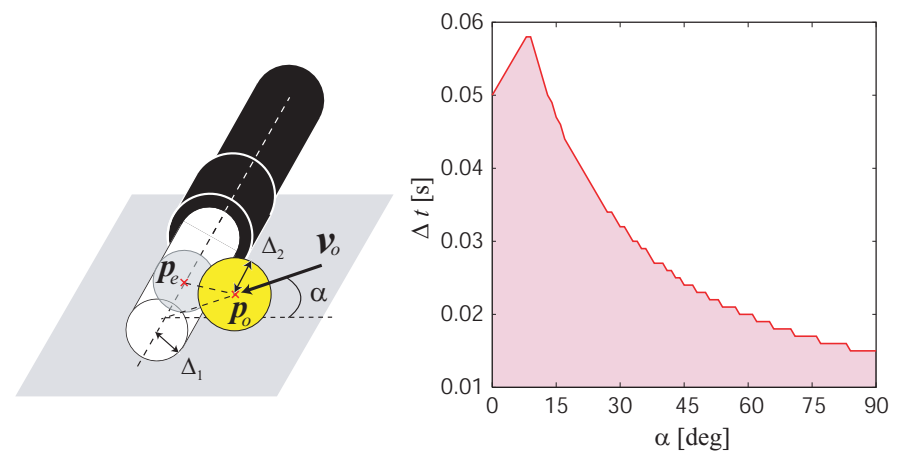

Fig. 11. The margin of time error

as short as possible. That is, arbitrary vectors $\boldsymbol{c}_{v}, \boldsymbol{c}_{a}$ are set by trial and error so that Eq.(19) is satisfied.

\section{Following capability for variation in target trajectory}

Suppose that at $t=t_{b}$ the ball reaches the plane expressed by Eq.(8), then we can examine how far the manipulator can follow the shift in hitting point on the plane during the swing. The time error is described in the IV-D.

In this experiment the moving velocity of the hitting point is under $2 \mathrm{~m} / \mathrm{s}$. Therefore we verify the following capability in the case of $\hat{\boldsymbol{p}}_{b}(t)=\hat{\boldsymbol{p}}_{b}(0)+\boldsymbol{v}_{b} t,\left\|\boldsymbol{v}_{b}\right\|=2 \mathrm{~m} / \mathrm{s}$. The result is shown in Fig.10. In the $z$ direction high and low hitting points $\hat{\boldsymbol{p}}_{b}(0)$ are written. The radiating lines represent movement of the hitting point. The possible area where the manipulator can follow the ball is saturated not by the limit of velocity or torque but by the structure of the manipulator or swing time. Moreover the similar results are obtained for other hitting points. That is, the batting task is achieved as long as the hitting point moves within the the following zone;

$$
\begin{aligned}
& W=\left\{\boldsymbol{p} \mid L_{f}-\frac{l}{2} \leq\left\|\boldsymbol{p}-\hat{\boldsymbol{p}}^{S W}\right\| \leq L_{f}+\frac{l}{2},\right. \\
& \left.p_{x}=\hat{\boldsymbol{p}}_{b}(0) \cdot \boldsymbol{e}_{x}\right\},
\end{aligned}
$$

where $l$ is the length of the bat. We verify that the manipulator can hit a ball that moves $40 \mathrm{~cm}$ in the radial direction during the swing.

\section{Hitting capability for time error}

To simplify the problem, the shape of the bat is taken as a cylinder and we discuss the margin of time error without

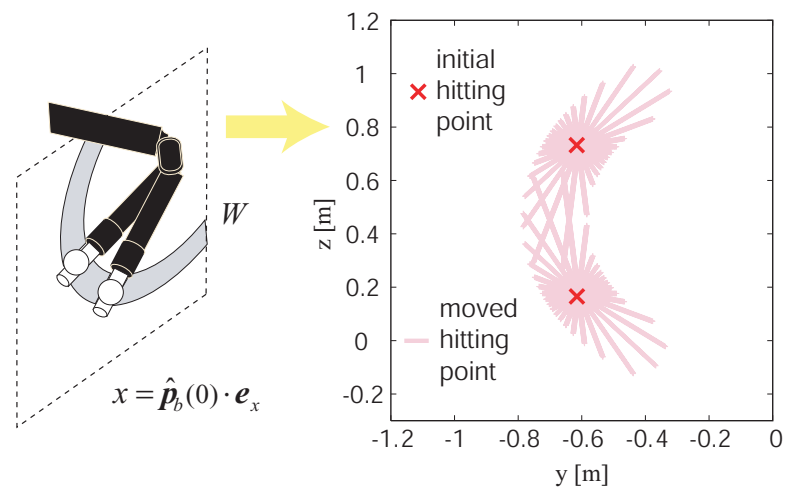

Fig. 10. Area where a manipulator can follow the shift in hitting point considering the direction of the ball after impact. We assume that the ball passes through the plane expressed by Eq.(8) at $t=t_{b}+\Delta t$. In this case, the impact condition between the bat with radius $\Delta_{1}$ and the ball with radius $\Delta_{2}$ is given as

$$
{ }^{\exists} \Delta t \text { s.t. }\left\|\boldsymbol{p}_{e}(t)-\boldsymbol{p}_{o}(t)\right\| \leq \Delta_{1}+\Delta_{2} .
$$

We calculate the margin of time error for uniform motion of a ball thrown from various angles into a fixed hitting point $\hat{\boldsymbol{p}}_{b}$. In Fig.11 the results are shown for the case of $\Delta_{1}=\Delta_{2}=5 \mathrm{~cm}$, $\boldsymbol{p}_{o}(t)=\hat{\boldsymbol{p}}_{b}+\boldsymbol{v}_{o}\left(t-t_{b}-\Delta t\right),\left\|\boldsymbol{v}_{o}\right\|=6 \mathrm{~m} / \mathrm{s}$. Horizontal axis $\alpha$ represents an incident angle for the horizontal plane at impact point. When the incident angle equals 9 degree, the margin of time error is a maximum of up to $58 \mathrm{~ms}$. This is because this angle is nearly parallel to the vector for the manipulator velocity at the impact point. We verified the manipulator can hit a ball with a time error of $23 \%$ of swing time $0.25 \mathrm{~s}$.

\section{CONCLUSION}

In this paper we proposed a hybrid trajectory generator that integrates two motions: mechanical high-speed motion and sensor-based reactive motion. In addition, the high-speed spray hitting task was achieved. Moreover we analyzed the proposed algorithm by computer simulation.

Our future work will concentrate on high-speed dexterous manipulation with hand-arm coordination.

\section{REFERENCES}

[1] M.T. Mason and K.M. Lynch. Dynamic Manipulation. Proc. IEEE Int. Conf. on Intelligent Robots and Systems, pp.152-159, 1993.

[2] S. Kawamura, W. Choe, S. Tanaka, and S.R. Pandian. Development of an Ultrahigh Speed Robot FALCON using Wire Drive System. Proc. IEEE Int. Conf. on Robotics and Automation, pp.215-220, 1995.

[3] M. Kaneko, M. Higashimori, R. Takenaka, A. Namiki and M. Ishikawa. The 100G Capturing Robot -too Fast to See-. IEEE Trans. on Mechatronics, Vol.8, No.1, pp.37-44, 2003.

[4] A. Namiki, and M. Ishikawa. Sensory-Motor Fusion Architecture Based on High-Speed Sensory Feedback and Its Application to Grasping and Manipulation Proc. 32nd Int. Symp. on Robotics, 2001.

[5] M. Bühler, D. Koditschek, and P. Kindlmann. Planning and control of robotic juggling and catching tasks. Int. J. of Robotics Research, 13(2), pp.101-118, 1994.

[6] T. Senoo, A. Namiki and M. Ishikawa. High-Speed Batting Using a MultiJointed Manipulator. Proc. IEEE Int. Conf. on Robotics and Automation, pp.1191-1196, 2004.

[7] Y. Nakabo, M. Ishikawa, H. Toyoda, and S. Mizuno. 1ms Column Parallel Vision System and It's Application of High Speed Target Tracking. Proc. IEEE Int. Conf. on Robotics and Automation, pp.650-655, 2000.

[8] http://www.k2.t.u-tokyo.ac.jp/fusion/SprayHitting/ 